\title{
Healthcare professionals' perceptions of privacy and dignity afforded to palliative care patients in an acute hospital setting: A qualitative descriptive study
}

\author{
Niamh Gantley ${ }^{1}$, Carmel Bradshaw ${ }^{2}$ and Owen Doody ${ }^{2 *}$ \\ ${ }^{1}$ Clinical Nurse Specialist, Galway University Hospital, Ireland \\ ${ }^{2}$ Department of Nursing and Midwifery, University of Limerick, Limerick, Ireland
}

\begin{abstract}
Background: Privacy and dignity play a fundamental and vital part of care for all patients, families and carers. However, healthcare professionals can be challenged in delivering privacy and dignity to palliative care patients in acute hospitals.

Objectives: To explore healthcare professionals' perspectives on maintaining and promoting privacy and dignity for palliative care patients in an acute health care setting.

Method: A qualitative descriptive study of fifteen participants. Following receipt of ethical approval, semi-structured interviews were audio recorded and transcribed. Participants included nurses and doctors with experience in palliative care. Data were analyzed using Burnard's framework for analysis.

Results: The findings of this study identify that healthcare professionals endeavour to do their best when caring for patients with palliative needs. However, in the context of current working environments, staff shortages and ward layout, privacy and dignity of patients are sometimes forgotten. Although health professionals understand the concepts of privacy and dignity and realise its importance for patients, they acknowledge that it's not always a high care priority and patient autonomy can be eroded in the acute setting. Healthcare professionals feel it is difficult to provide privacy and dignity to palliative care patients in the acute hospital setting and highlight the challenges.

Conclusion: Healthcare professionals are aware that there are many limitations to the quality of privacy and dignity afforded to patients, despite their best efforts. However, privacy and dignity need to be given a higher priority by health professionals. All health professionals need further education and the culture among staff members needs to change to support palliative care patients to have more control over their environment and their medical care. All of these have resource issues and acute hospitals also need more financial support to change infrastructure to promote and implement privacy and dignity for all patients.
\end{abstract}

\section{Introduction}

The word dignity comes from the Latin word dignus (worth) or dignitus which means (merit) while the word privacy comes from the Latin word privitas and privo meaning 'to deprive'. These can be difficult concepts to define [1] but providing privacy and dignity to patients is a fundamental aspect of providing effective palliative care [2]. Privacy and dignity are basic human needs, and every patient has the right to experience them and all healthcare professionals have an obligation to provide privacy and dignity to patients. It is important to address privacy and dignity as these values can be weakened when a patient is unwell and vulnerable. It is recognised that if dignity is upheld it merges with many features of privacy such as; respecting the person, keeping their body private and having control over their own space, and in preserving dignity patients are able to live in accordance with their morals and values, be treated as an equal and be respected as a person [3]. The fundamentals of palliative care involve providing patients with privacy and dignity, however, patients' perceptions of what privacy and dignity are for them must first be understood $[4,5]$. Each person has an individual experience at end-of-life [6] and differences exist in how people perceive their patient experiences [7]. Privacy and dignity affect one's experience of end-of-life care, with patients who have died in private rooms been perceived as having better end-of-life care. Furthermore, dependence level has been identified as a factor impacting on personal dignity and dignity is perceived as been more likely violated in a hospital setting [8].

The experience of end-of-life care can be positive, when mutual understanding is achieved. Patients identify they want staff to have a greater awareness of them, take the time to talk, to offer flexibility and promote individuality and where this is the case staff have reported been able to better understand patients and deliver more appropriate care [2]. Through taking time and working with patients, patients can be treated with respect, be viewed as the person they are, told the truth, have their symptoms controlled and not prolong their suffering if the end is inevitable, supported to maintain control and choice until the end-of-life and to have privacy [9]. However, in spite of this knowledge there is a reported lack of education and knowledge regarding the importance of maintaining privacy and dignity and this is a barrier to

${ }^{*}$ Correspondence to: Owen Doody, Department of Nursing and Midwifery, University of Limerick, Limerick, Ireland, V94 T9PX, E-mail: owen.doody@ul.ie

Key words: dignity, privacy, palliative care, acute hospital, nursing

Received: September 22, 2020; Accepted: September 29, 2020; Published: October 05,2020 

study

providing the best possible care to patients at end-of-life [8]. A lack of awareness and training may result in breaches to dignity of older patients [10] and dying with dignity is a human right where dignity includes patients having privacy and a quiet environment [11]. However, within the busy environment of acute hospitals, health care professional can have difficulties in maintaining privacy and dignity of dying patients [12]. This paper explores healthcare professionals' perspectives on maintaining and promoting privacy and dignity for palliative care patients in an acute healthcare setting.

\section{Methods}

A qualitative design was chosen to address the aim of this study as it combines the scientific and artistic nature of nursing to enhance understanding of the human health experience [13]. Thus, enabling the researchers discover the complexity of healthcare professionals' perspectives in maintaining and promoting privacy and dignity for palliative care patients in an acute health care setting. The study utilised a qualitative descriptive design described by Bradshaw et al., [14] as information was required directly from those experiencing the phenomenon or lived experience providing a vehicle for the voices of those experiencing the phenomena of interest which "can transform nursing and midwifery practice and indeed health care services generally" [14]. Ethical approval was gained from the hospital research ethics committee and access through the director of nursing and the medical director of the hospital. Two units within an acute hospital were chosen as both units have 25 oncology/palliative care patients, similar staffing levels, palliative care workload, and physical layout. Prior to data collection an interview guide was developed based on a review of the literature [15]. Three pilot interviews were undertaken with nurses who had palliative care experience but were not part of the study sample, to inform the researchers about the suitability of the interview guide and questions. The interviews followed a semi structured format and were audiotaped for transcription purposes and all participants were offered the opportunity to review a copy of their transcript. Participants comprised of 10 nurses (5 from each unit) and 5 doctors who work in palliative care. Thematic analysis was conducted following Burnard [16] framework of; taking memos after each interview, reading transcripts and making notes of general themes, repeated reading and generating open coding headings to describe all aspects of the data, reducing the codes under higher order headings, returning to the data with the higher order codes and collating the organised data for reporting.

\section{Findings}

Through data analysis three themes were identified which were: Doing our best, Stolen autonomy and Education and training. All fifteen of the participants were female and of the 10 nurses all had palliative care experience ranging from 3 to 22 years with an average of $13^{1 / 4}$ years and for the 5 doctors palliative care experience ranged from $1^{1 / 2}$ years to 17 years with an average of $7^{3 / 4}$ years.

\section{Doing our best}

A common theme voiced by participants was that they 'tried their best', "nurses try their best but I suppose we could do better... I think the staff on the wards and even the more junior doctors are trying to provide privacy and dignity, but I just don't think it is getting ticked off because there are too many interruptions, like it's the acute setting" (P4).

This sense of trying their best was common among participants, "we certainly try to do our best, I feel sometimes that I do a good job when patients pass away peacefully and comfortable ... we try to manage patients care as best as we can" (P7) although "it is not always possible to carry it out fully but where and when, we do try" (P 10).

However, participants also stated that they are not very good at promoting privacy and dignity and it "falls down the priority list" (P2) but "we try our best to get patients a single room if they are dying" (P9). Conversely, some participants felt that, "people don't expect privacy in an acute hospital" (P1) and "it's not on some healthcare professional's radar" (P9).

Many participants felt there should be, "no variance in care for a palliative care patient in comparison to any other patient in the hospital as all patients deserve the same treatment" (P3, P9, P13). However, some participants felt that, "palliative care patients deserved more privacy and dignity" (P2, P12).

One means identified to support the privacy and dignity was the, "ability to have a single room" (P2, P6, P7, P10), and to ensure "activities of daily living in private surrounds" (P1, P3, P9), "without interruptions" (P1, P4, P13, P14, P15), "ensuring patients had control over their environment" (P11, P12) and that health professionals "respected what the patient wanted" (P1, P8, P13, P15).

However, "that doesn't always happen as we would have a lot of palliative care patients but patients that are dying, we would always have them in a single room and for their families" (P6).

Interestingly it was identified that not all patients wish to be in a single room and their choice needs to be ascertained and respected, "I had a patient who did not feel comfortable to be in a single room, his daughter had passed away in a single room and we had to respect his decision" (P4). As part of respecting individual patient choices nurses reported they need to advocate for patients and their families and be confident in challenging doctors on their medical choices and that, "with experience she gained confidence" (P7)

\section{Stolen autonomy}

Frequently, healthcare professionals suggested that patients have limited control over their space and that a patients' area or space within an acute hospital is viewed as an "open house" (P9). This was seen as an issue as patients and families have no way of preventing interruptions even in single rooms and patients have no control of their own time, "it's a kind of open house for any staff member, it doesn't matter who you are, kitchen staff or nurse or doctor, if the curtains are drawn or door closed nobody knocks" (P9), "there are so many professionals involved in care of the patients and they are all in a rush and they need to speak to patients, so patients could literally see someone on an hourly basis whether they are eating their lunch, whether they are on the loo, and even then someone waits for them to finish on the loo outside the door and when they come out then straight away someone is asking them questions and I think it's such a busy environment that privacy and dignity is probably not always our priority...in the acute setting people come and go all day, there are not set times" $(\mathrm{Pl})$

Both nurses and doctors mentioned that, "it is very difficult to know when you can't go into to a patient if there is private care going on, you have no way of knowing, nurses acting as advocates is essential" (P11) and that "patients don't realise you are coming in until you are around the bed and then you're having to apologise profusely, if the patient is in the middle of toileting, I suppose where is the protected time to get these things done, it's not getting the recognition it deserves" (P11). 

study

Likewise, it was noted that, "it's very frustrating you would be in the middle of a very sensitive conversation with a patient about death and dying and the cleaners walk in to clean the room, or other medical teams who take over" (P15).

Participants reported repeatedly that patients and their families have little if no control over traffic into their private space. Even with signs on doors explicitly asking for no visitors or interruptions, the culture seems to be that staff and visitors have a right to enter. Often, "visitors just looked in all the single room windows until they found the person they were looking for" (P5) and even when "we put the light system on outside the door to alert people that care is in progress, $90 \%$ of the time nobody took any notice" (P10)

Thus, reinforcing that although mechanisms exist to prevent people from going into the rooms of patients at vulnerable times, this is not adhered to. In the acute hospital setting patient autonomy is also affected as, "there is so much emphasis on the medical side rather than the personal side" (P5), emphasised by "looking through most medical charts there is never once a mention of what the patient wants in relation to treatment or choice" (P15).

This lack of focus on the patient's needs may be due to healthcare professionals trying to maintain control or more likely avoiding difficult situations, "we can be very afraid to talk about death and dying and it would be no harm to discuss their wishes and their wants when they are well" (P11) and "I wonder sometimes do we forget that dying is inevitable, and we can treat illness for whatever length of time but ultimately its limited and I am not sure that in general we are good at talking about death and dying or to encourage patients to consider it" (P15).

In addition, patients' autonomy can be further eroded, "some patients are unaware what doctors are discussing with family members" (P9). Furthermore, the drive for a medical approach can cause conflict and distress and "sometimes you have to pull back and try and let the person have their dignity and respect prior to dying and then the oncology team come in and go, no we will go down and get the $x$-ray, you have to be strong and say no and this would happen often being honest" (P4).

The issue of time was a common concern, and that the very nature of palliative care patient means that time is precious and the time spent with families and loved ones is important "it's their time, it's their families time and it's the little disturbances that cause harm" (P7). Furthermore, privacy needs to be "understood from the patient's point of view" (Pl) and subtle suggestions need to be considered like when "patients express their concern that they can't get sleep and rest" (P8) or "we can be bad, we are just getting the job done and don't give patients the time, we need to ask the patient is it a good time to come and see you" (P2) as privacy and dignity "can be tricky" (P5).

\section{Education and Training}

Participants felt that more training and education was required to help improve standards in palliative care. Promoting privacy and dignity was vital to enhance this aspect of care, "I think the promotion of privacy and dignity would be a good idea, I think it's more relevant than we really appreciate, and to promote it would make a bigger difference to the patients than we may think but like there are lots of like infection control and hand hygiene that we all have to do, so for privacy and dignity there should be something we all have to do... it would give a great boost to the area" (Pl).

Additionally, it was noted that there is not enough emphasis on privacy and dignity training at an organizational level and that staff don't get the opportunity to access relevant education which needs to be embedded into the culture of the organisation, "I don't get any specific training on how to deal with end of life... you know say there is no ongoing training and it can be quite hard how do you know, there's communication skills, how do you give news, privacy and dignity at end of life specifically" (P9) and "you need organisational changes in terms of culture and I think that would be very much be part of specialist training whether it would be on rounds or you know practical sessions ward by ward" (Pl l). Moreover, participants felt that there should be a practical approach to education and training "show how it (privacy and dignity) is actually implemented or practical ways of promoting the privacy and dignity for the patient so education and the practical steps to implement $i t^{\prime \prime}(\mathrm{Pl} 2)$.

Of the fifteen participants, eight of them were unaware there was a privacy and dignity policy in a large acute university hospital. A further four reported that they knew there was a policy, but they didn't know what the policy entailed and only two participants reported familiarity with the details of the privacy and dignity policy. Eleven of the fifteen participants had experiences of patients' dissatisfaction with the maintenance of their privacy and dignity in the acute hospital setting. Ten participants felt that the standards of patients' privacy and dignity had actually improved over the past ten years but that there was still a lot of scope for improvement. Of interest, participants noted that through engaging with this study it brought the topic to their minds and caused them to question and reflect on their own practice, "I just think it's not until its highlighted and somebody coming and asking you questions like this, that it becomes a little bit more paramount in your head and that you would be able to bring it back into your practice and go hang on actually we need to $x$ or $y^{\prime \prime}(\mathrm{P} 4)$.

\section{Discussion}

This study reinforces Whitehead and Wheeler [17,18] focus on patients' privacy and dignity including being treated in private spaces, having one's own space and being treated as a person with sympathy and compassion. Likewise, the findings of this research highlights healthcare professionals perceptions of how privacy and dignity should be afforded to palliative care patients in the acute hospital. Privacy and dignity which includes awareness, environmental, cultural and education aspects need to be addressed at an individual, professional and organizational level. The concepts of privacy and dignity are interlinked, and this is reiterated in this study where it was felt that privacy and dignity were "interconnected but quite separate". The importance of, and the obligation to maintain privacy and dignity for patients are represented in both nurses and doctors' codes of ethics. However, from this study there are many examples of scenarios where these basic human needs are simply not met for palliative care patients. Examples include nurses being in the middle of sensitive care for patients with members of the medical team interrupting that care or when a doctor has gone to examine a patient and mid conversation realises that the patient is on a bed pan. This highlights that such significant values can be weakened when patients are unwell and at their most vulnerable and are often overlooked as a priority by health professionals and in fact sometimes completely neglected in the drive to "get the job done" $[2,3]$.

Nurses and doctors in this study indicated that they do 'try their best' and especially with end-of-life patients, but acknowledged that this can be difficult with the acuity of the setting, shortages in staff and the general unit/ward layout. This is concerning for future patient care as Kane et al. [19] predicts that there will be a significant increase in 

study

patients diagnosed with life limiting illnesses such as cancer, dementia and other terminal illnesses. Therefore, increased numbers of palliative care patients are to be expected within the acute hospital setting. Given that this study illustrates that health professionals in the context of current working conditions are trying their best, but despite this privacy and dignity are often seen as a lower priority in comparison to other aspects of their care does not augur well for future health care provision for this very vulnerable cohort of patients. Support is needed for staff in the form of education and training as a matter of urgency in tandem with addressing environmental conditions unfavourable to maintaining privacy and dignity and privacy for patients requiring end of life care [19]. In the absence of such supports and the drive for person-centred approach to care with a focus on the patient experience, health professionals may experience greater stress, exhaustion, depersonalisation and burnout [20].

Every person deserves a dignified death [21], this study highlights that although staff try to get it right, they don't always succeed, and sometimes medical care can override or dominate the reality of what's happening. The key to effective person-centred care is knowing when to pull back and facilitate dignity and respect for the person prior to their dying [22]. Within this study it was apparent that patients had little control in relation to timing, hospital routines and consultations and having a single room did not mean a patient would be free from such interruptions [23]. To address such issues a change in culture is necessary. This is not an easy process as is apparent in this study where measures put in place to protect privacy and dignity were ignored. To support a cultural change, continuous promotion and education is warranted in the acute hospital setting where the need for change and how to effect change should be discussed [24] and education and training available [8]. It has been maintained by Chocinov et al., [25] that patients and families experiencing "detached automatic healthcare and that patient hood trumps personhood and that caring remains carer's distant cousin", sadly mirrored in this study's findings. On the evidence of this study, it cannot be denied that patients' privacy and dignity may be overlooked in the acute hospital setting. Although improvements in patients' privacy and dignity have been made, for example with hospice friendly hospitals project, palliative care for all and design and dignity programmes, more needs to be done. This includes addressing awareness, culture, environment, professional and education and training needs of all hospital staff to ensure that privacy and dignity are afforded priority in the care of all patients, but particularly those at the end of life.

\section{Conclusion}

This study highlights that privacy and dignity need attention in practice and particularly within the acute setting for patients with palliative care needs. A change in staff culture through education, training and environmental supports is warranted and hospital management need to take responsibility for how hospitals address this issue. For this to occur there needs to be more open discussions on death and dying involving patients and their families to ensure their needs are heard and met, and greater openness and communication across all hospital workers which includes ancillary staff such as porters, cleaners and catering. In addition, while all hospitals may have policies on privacy and dignity, there is a need to promote awareness of the existence of such policies and support through specifically assigned staff to champion privacy and dignity at a unit/ward level. While this study gives us insight into healthcare professionals perceptions of privacy and dignity in practice, patient or family perceptions were not gained which would be very valuable. In addition, participants in the study had an extensive experience of palliative care in their areas and findings may differ if the study was undertaken throughout the whole of the acute setting, particularly where palliative care is less commonly experienced.

\section{References}

1. Mozaffari N, Dadkhah B, Payandeh H (2019) Comparing perceived dignity and privacy in patients hospitalized in internal and surgical wards of Ardabil educational medical centers. J Adv Pharm Educ Res 9 S2: 39.

2. Johnston B, Pringle J, Gaffney M, Narayanasamy M, McGuire M, Buchanen D (2015) The dignified approach to care: a pilot study using the patient dignity question as an intervention to enhance dignity and person centred care for people with palliative care needs in the acute hospital setting. BMC Palliat Care 14: 1-14. [Crossref]

3. Barclay L (2016) In sickness and in dignity: A philosophical account of themeaning of dignity in health care. Int J Nurs Stud 61, 136-141. [Crossref]

4. Guo Q, Zheng R, Jacelon CS, McClement S, Thompson G, et al. (2019) Dignity of the patient--family unit: further understanding in hospice palliative care. BMJ Support Palliat Care 2019. [Crossref]

5. Svensson G, Wahlin I (2018) Patient perceptions of specialised hospital-based palliative home care: a qualitative study using a phenomenographical approach. Int J Palliat Nurs 24: 22-32. [Crossref]

6. Reed FM, Fitzgerald L, Bish MR (2017) Rural district nursing experiences of successful advocacy for person-centered end-of-life choice. J Holist Nurs 35: 151164. [Crossref]

7. Migala S, Flick U (2019) Cultural and individual barriers to palliative care from different angles: Data triangulation in practice. Qualitative Inquiry 25: 786-798.

8. Pringle J, Johnston B, Deans B (2015) Dignity and patient centred care for people with palliative care needs in the acute hospital setting: A systematic review. Palliat Med 29: 675-694. [Crossref]

9. Irish Hospice Foundation (2017) Have Your Say Initial analysis of data from a nationa survey to inform the development of an Irish Charter on Death, Dying and Bereavement in Ireland. Irish Hospice Foundation. Dublin.

10. Tauber- Gilmore M, Addis G, Zahran Z, Black S, Baillie L, Proctor S, Norton C (2018) The views of older people and health professionals about dignity in acute hospital care. J Clin Nurs 27: 223-234. [Crossref]

11. Becker CA, Wright G, Schmit K (2017) Perceptions of dying well and distressing death by acute care nurses. Appl Nurs Res 33: 149-154. [Crossref]

12. Cagle JG, Unroe KT, Bunting M, Bernard BL, Miller SC (2017) Caring for dying patients in the nursing home: Voices from frontline nursing home staff. J Pain Symptom Manage 53: 198-207. [Crossref]

13. Newell R, Burnard P (2011) Research for evidence-based practice in healthcare. $2^{\text {nd }}$ edition. Oxford: Blackwell Publishing.

14. Bradshaw C, Atkinson S, Doody O (2017) Employing a Qualitative Description Approach in Health Care Research. Glob Qual Nurs Res 4. [Crossref]

15. Doody O, Noonan M (2013) Preparing and conducting interviews to collect data. Nurse Researcher 20: 28-32. [Crossref]

16. Burnard P (2006) A Pragmatic approach to qualitative data analysis. In: Newell, R, Burnard, P. Research for evidence-based practice in healthcare. $2^{\text {nd }}$ edition, pp.118-129. Oxford: Blackwell Publishing.

17. Whitehead J, Wheeler H (2008a) Patients' experiences of privacy and dignity Part 1: A literature review. Br J Nurs 17: 381-385. [Crossref]

18. Whitehead J, Wheeler H (2008b) Patients' experience of privacy and dignity Part 2: An empirical study. Br J Nurs 17: 458-464. [Crossref]

19. Kane P, Daveson B, Ryan K, McQuillan R, Higginson I, Murtagh F (2015) The need for palliative care in Ireland: a population-based estimate of palliative care using routine mortality data, inclusive of nonmalignant conditions. J Pain Symptom Manage 49: 726733. [Crossref]

20. de Paiva LC, Canário ACG, de Paiva China ELC, Monteiro BR, da Silveira AKG (2016) Risk factors identification for Burnout syndrome in nurses. International Archives of Medicine, 9.

21. Mason H, Derubeis M, Mason N, Worden F (2018) Ethical Considerations in End-of Life Discussions. J Nurs Healthcare 3: 1-7. 
Doody O (2020) Healthcare professionals' perceptions of privacy and dignity afforded to palliative care patients in an acute hospital setting: A qualitative descriptive study

22. Hahn AL (2020) Transitioning from Curative Care to End-of-Live Care in the Acute Care Hospital: A Hermeneutical Phenomenological Study. Doctoral dissertation, Faculty of Graduate Studies and Research, University of Regina.

23. Hosseini FA, Momennasab M, Yektatalab S, Zareiyan A (2019) Patients' perception of dignity in Iranian general hospital settings. Nurs Ethics 26: 1777-1790. [Crossref]
24. McCourt R, Power JJ, Glackin M (2013) General nurses' experiences of end-of-life care in the acute hospital setting: a literature review. Int J Palliat Nurs 19: 510-516. [Crossref]

25. Chochinov H, McClement S, Hack T, Thompson G, Dufault B, et al. (2015) Eliciting personhood within clinical practice: Effects on Patients, Families, and Health Care Providers. Journal of Pain and Symptom Management 49: 974-980. [Crossref]

Copyright: (C2020 Doody O. This is an open-access article distributed under the terms of the Creative Commons Attribution License, which permits unrestricted use, distribution, and reproduction in any medium, provided the original author and source are credited. 